14,07

\title{
Особенности определения модуля упругости сверхориентированных высокопрочных пленочных нитей, полученных вытяжкой ксерогелей сверхвысокомолекулярного полиэтилена
}

\author{
(С) Ю.М. Бойко ${ }^{1}$, В.А. Марихин ${ }^{1}$, О.А. Москалюк ${ }^{2}$, Л.П. Мясникова ${ }^{1}$ \\ ${ }^{1}$ Физико-технический институт им. А.Ф. Иофрфе РАН, \\ Санкт-Петербург, Россия \\ ${ }^{2}$ Санкт-Петербургский государственный университет технологии и дизайна, \\ Санкт-Петербург, Россия \\ E-mail: yuri.boiko@mail.ioffe.ru
}

(Поступила в Редакцию 11 июля 2018 г.)

Исследовано влияние рабочей длины образца $l_{0}$ на величину модуля упругости $E$ при растяжении сверхориентированных пленочных нитей сверхвысокомолекулярного полиэтилена (СВМПЭ). Образцы нитей были получены методом многостадийной ориентационной вытяжки на контактном нагревателе с достижением очень высоких значений степени вытяжки $\lambda=120$. В качестве прекурсора использовались ксерогели, полученные сушкой 1.5\%-ых гель-растворов СВМПЭ в декалине. Выявлено существенное увеличение „методической“ величины $E$ от 100 до $230 \mathrm{GPa}$ при увеличении значения $l_{0}$ от 10 до $80 \mathrm{~mm}$, имеющее затухающий характер. Показано, что зависимость $E$ от $l_{0}$ может быть описана с помощью эмпирического уравнения, позволяющего осуществлять прогноз величины $E$ в зависимости от $l_{0}$. В соответствии с прогнозом насыщение истинной величины $E$ с выходом на плато $E=242 \mathrm{GPa}$ происходит при $l_{0}=200 \mathrm{~mm}$.

Работа выполнена при финансовой поддержке РФФИ, проект № 18-29-17023мк.

DOI: 10.21883/FTT.2019.01.46911.207

\section{1. Введение}

Получение высокопрочных высокомодульных полимерных материалов является перспективным направлением в области синтеза и переработки полимеров, направленным на расширение спектра изделий с очень высокими механическими характеристиками - прочностью $\sigma_{b}$ и модулем упругости $E$. Одним из таких материалов являются ультраориентированные пленочные нити из сверхвысокомолекулярного полиэтилена (СВМПЭ, с молекулярной массой $M>10^{6} \mathrm{~g} / \mathrm{mol}$ ), полученные в лабораторных условиях методом высокотемпературной зонной многоступенчатой ориентационной вытяжки ксерогелей СВМПЭ, разработанным в ФТИ им. А.Ф. Иоффе [1-3]. Полученные мононити удавалось вытянуть до весьма высоких значений степени вытяжки $\lambda=120$. В результате были достигнуты очень высокие значения $\sigma_{b}=4.7 \mathrm{GPa}[2]$, существенно превышающие прочность $\sigma_{b}=3.6 \mathrm{GPa}$ сверхориентированных промышленных волокон Dyneema (DSM, the Netherlands) и Spectra (Honeywell, USA), также сформованных из гелей СВМПЭ [4,5]. Причем для 20\% образцов впервые были зарегистрированы достоверные сверхвысокие значения $\sigma_{b}=5.5-6.5 \mathrm{GPa}$ по результатам статистического анализа большого массива данных (пятидесяти измерений идентичных образцов).

Наряду с прочностью, не менее важной механической характеристикой является модуль упругости $E$, поскольку он определяет возможность сохранения формы изделий при их эксплуатации. Кроме того, высокомодульные материалы, к числу которых относится и высокоориен- тированный СВМПЭ, обладают свойством диссипации энергии от места удара по объему образца, что является важным при их использовании в качестве элементов бронезащиты. Поэтому в данной работе основное внимание уделяется исследованию модуля упругости ультраориентированных пленочных нитей СВМПЭ.

Обычно для измерения величины $E$ используется наиболее простой и доступный способ измерения по наклону начального участка кривой деформирования $(E=d \sigma / d \varepsilon)$ при использовании деформации $\varepsilon$, измеренной между зажимами разрывной машины. Вместе с тем, более корректным подходом для определения величины $E$ является использование ,истинной“ деформации (а следовательно и истинного модуля упругости), измеренной при использовании экстензометров (например, щуповых датчиков деформации фирмы Zwick или навесных датчиков деформации фирмы Instron), закрепленных на рабочей части (базе) образца. Действительно, в этом случае исключается нежелательное выскальзывание образца из зажимов, вносящее погрешность в измерение модуля упругости. Однако этот подход оказывается проблематичным применительно к ультраориентированным пленочным нитям по следующим причинам. Во-первых, при закреплении (в ряде случаев, достаточно массивных по отношению к массе образца) датчиков деформации возможен прогиб таких образцов в силу их очень маленького поперечного сечения (порядка $10^{-9} \mathrm{~m}^{2}$ ), что вносит искажение в регистрацию кривой деформирования. Во-вторых, одним из больших недостатков является низкий коэффициент трения полиэтиленовых материалов, в особенности высокоориентированных, что 
приводит к проскальзыванию элементов крепления экстензометра на поверхности образца.

Таким образом, целью настоящей работы является поиск надежного способа определения модуля упругости ультраориентированных пленочных нитей СВМПЭ при использовании деформации, измеренной между зажимами механического тестера.

\section{2. Экспериментальная часть}

Навеску реакторного порошка СВМПЭ со средневязкостной молекулярной массой $M_{\eta}=3 \cdot 10^{6} \mathrm{~g} / \mathrm{mol}$, синтезированного на нанесенных титан-магниевых катализаторах в Институте катализа им. Г.К. Борескова СО РАН, Новосибирск, помещали в пробирку с растворителем (декалин) и антиоксидантом (0.1 wt.\% ди-трет-бутил- $n$ крезола) для получения $1.5 \%$-го раствора полимера. Пробирку погружали в термостат, нагретый до температуры $T=100^{\circ} \mathrm{C}$, и затем повышали $T$ до температуры растворения СВМПЭ $\left(120^{\circ} \mathrm{C}\right)$ при постоянном перемешивании. Перемешивание прекращали при резком увеличении вязкости раствора, извлекали мешалку и вставляли в пробирку обратный холодильник с притертой пробкой. Затем раствор нагревали до $T=160^{\circ} \mathrm{C}$ и выдерживали при этой температуре в течение $1 \mathrm{~h}$ для достижения его гомогенизации, после чего прозрачный однородный раствор выливали в чашку Петри при комнатной температуре. За счет резкого охлаждения (от 160 до $20^{\circ} \mathrm{C}$ ) и последующей кристаллизации раствор переходил в состояние жидкого геля с образованием пористой структуры из хаотически ориентированных взаимосвязанных стопок ламелей (типа губчатой структуры). После высушивания „мокрых гелей“ получали так называемые ксерогели - твердые непрозрачные тонкие пленки толщиной порядка $100 \mu \mathrm{m}$. Высушенные пленки нарезались на узкие полоски шириной около $1 \mathrm{~mm} \mathrm{c}$ помощью специально изготовленного приспособления, которые и подвергались многоступенчатой ориентационной вытяжке на лабораторной установке по методу [1]. Изменяя ориентирующее усилие и ступенчато повышая температуру контактного нагревателя вплоть до $139-141^{\circ} \mathrm{C}$, то есть до температуры плавления ПЭ в ненагруженном состоянии, были получены образцы пленочных мононитей СВМПЭ с очень высокой кратностью (степенью) вытяжки $\lambda=120 \pm 10$. Полученные образцы деформировались на универсальной разрывной машине Instron-1122 при скорости растяжения $10 \mathrm{~mm} / \mathrm{min}$ и комнатной температуре. База образца (расстояние между зажимами) $l_{0}$ изменялась от $10 \mathrm{~mm}$ (в соответствии с ГОСТ 10213.2-2002 [6]) до $l_{0}=80 \mathrm{~mm}$. Для предотвращения выскальзывания концов пленочной нити из зажимов и фиксации базовой длины $l_{0}$ образец вклеивался в бумажную рамку, продольная часть которой перерезалась перед проведением механических испытаний. Длина закрепленной в зажиме части образца $\left(l_{\text {clamp }}\right)$ во всех случаях составляла $10 \mathrm{~mm}$. Результаты механических характеристик были получены усреднением измерений пятидесяти $\left(l_{0}=10 \mathrm{~mm}\right)$, двенадцати $\left(l_{0}=50 \mathrm{~mm}\right)$ и трех $\left(l_{0}=30,40\right.$ и $\left.80 \mathrm{~mm}\right)$ идентичных образцов.

\section{3. Результаты и их обсуждение}

На рис. 1 приведены кривые растяжения для образцов высокоориентированной пленочной нити СВМПЭ с очень высокой $\lambda=120$ для нескольких значений $l_{0}$ в интервале от 10 до $80 \mathrm{~mm}$. Видно, что при увеличении значения $l_{0}$, с одной стороны, величина прочности $\sigma_{b}$ не зависит от $l_{0}$, являясь при этом весьма высокой $\left(\sigma_{b} \approx 4.7 \mathrm{GPa}\right)$. Факт слабой зависимости $\sigma_{b}$ от $l_{0}$ может быть объяснен тем, что максимальное изменение скорости деформации в исследованном интервале $l_{0}$ является сравнительно небольшим, трехкратным, в то время как заметное изменение $\sigma_{b}$ полимера ожидается при изменении скорости деформации на один десятичный порядок и больше [7]. С другой стороны, кривая деформирования в области малых $\varepsilon$ становится заметно круче, что приводит к резкому увеличению модуля упругости. Характерно, что наибольший эффект наблюдается при переходе $l_{0}$ от 10 до $30 \mathrm{~mm}$. Мы полагаем, что такое поведение может быть обусловлено вкладом деформации в зажимах в деформацию рабочей части образца, так как выскальзывания из зажимов исследованных образцов не происходило, что подтверждается формой кривой деформирования (монотонное возрастание напряжения с деформацией вплоть до разрушения образца нити во всех рассмотренных случаях). Действительно, образец пленочной нити (полоска) имеет одно и то же поперечное сечение как в зоне между зажимами, то есть в рабочей части, так и в самих зажимах. По этой причине

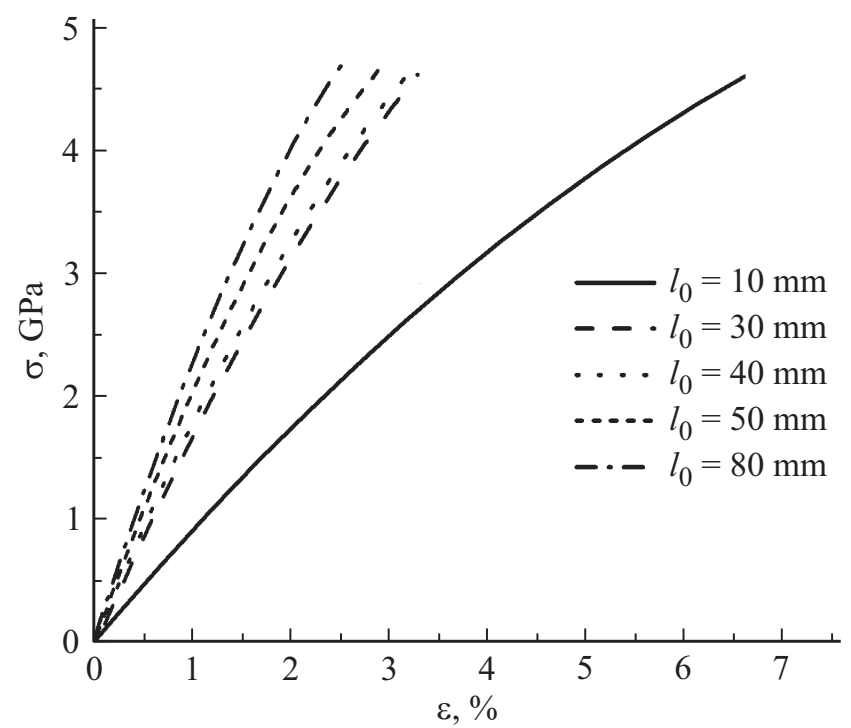

Рис. 1. Кривые растяжения ультраориентированных пленочных нитей на основе СВМПЭ с $\lambda=120$, сформованных в лабораторных условиях из $1.5 \%$-го раствора в декалине, при различной базовой длине образца $l_{0}$. 


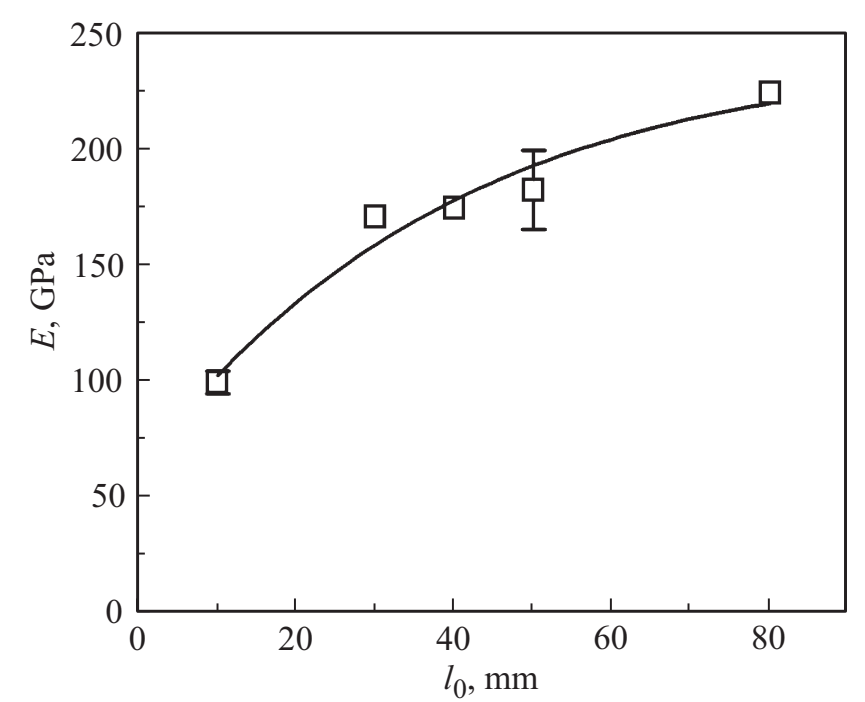

Рис. 2. Зависимость модуля упругости $E$ от расстояния между зажимами $l_{0}$ для лабораторных образцов ультраориентированных пленочных нитей на основе СВМПЭ с $\lambda=120$, сформованных из 1.5\%-го раствора в декалине; сплошная линия проведена в соответствии с уравнением (1).

удлинение образца под воздействием растягивающей силы должно осуществляться как между зажимами (удлинение $\Delta l$ на базе образца $l_{0}$ ), так и внутри каждого из двух зажимов (удлинение $\Delta l_{\text {clamp }}$ на базе $l_{\text {clamp }}$ ) за счет „вытекания“ материала из зажимов в рабочую часть. Причем с учетом напряжения сжатия в зажимах деформация закрепленной в зажимах части образца может быть даже большей, чем в его рабочей части. Следовательно, в силу вышеприведенных аргументов величина $E$, рассчитанная в области квазилинейной вязкоупругости $(\varepsilon \leq 1 \%)$, будет определяться не только относительным удлинением рабочей части образца $\Delta l / l_{0}$ как $E=\sigma /\left(\Delta l / l_{0}\right)$, где $\sigma-$ напряжение, но и относительным удлинением в двух зажимах $2\left(\Delta l_{\text {clamp }} / l_{\text {clamp }}\right)$, то есть в итоге как $E=\sigma /\left(\Delta l / l_{0}+2 \Delta l_{\text {clamp }} / l_{\text {clamp }}\right)$.

В таком случае для образца в форме полоски с $l_{0}=10 \mathrm{~mm}$, закрепленного в зажимах на длину $2 l_{\text {clamp }}=20 \mathrm{~mm}$, ожидаемое занижение $E$ составит $\left(l_{0}+2 l_{\text {clamp }}\right) /\left(l_{0}\right)=3$, тогда как при $l_{0}=80 \mathrm{~mm}$ оно составит всего лишь 1.25. Другими словами, в соответствии с проведенной оценкой при уменьшении $l_{0}$ от 80 до $10 \mathrm{~mm}$ происходит существенное занижение величины $E-$ в 2.4 раза. Такой „неожиданный“ эффект резкого методического уменьшения $E$ особенно выражен в сверхпрочных материалах. Он может приводить к неверной трактовке направления поиска путей дальнейшего увеличения $E$, хотя очень высокие значения $E$ уже достигнуты, но надежно не измерены.

С целью выяснения корректности оценки зависимости $E$ от $l_{0}$, проведенной выше, по наклону начальных участков кривых растяжения, приведенных на рис. 1, были рассчитаны значения $E$, которые приведены на рис. 2, в зависимости от $l_{0}$. Видно, что величина $E$ существенно увеличивается при увеличении $l_{0}$, и уже при $l_{0}=30 \mathrm{~mm}$ является весьма высокой, составляя $E=170 \mathrm{GPa}$ по сравнению с $E=99 \mathrm{GPa}$ при минимальной исследованной $l_{0}=10 \mathrm{~mm}$. При увеличении же $l_{0}$ во всем исследованном интервале, от 10 до $80 \mathrm{~mm}$, то есть в 8 раз, значение $E$ увеличивается от 99 до $225 \mathrm{GPa}$, то есть в 2.3 раза, что совпадает с выше проведенной теоретической оценкой (увеличение в 2.4 раза). Таким образом, полученный результат свидетельствует о справедливости предложенного подхода определения величины $E$ ультраориентированных образцов на основе СВМПЭ, учитывающего деформацию образца в зажимах.

Необходимо отметить, что выше определенное значение $E=225 \mathrm{GPa}$ для ультраориентированного СВМПЭ с $\lambda=120$ на один десятичный порядок превышает значение $E=22 \mathrm{GPa}$ для ориентированного высокомолекулярного полиэтилена (ПЭ) с молекулярной массой $M \sim 10^{5}$, полученного также методом многоступенчатой зонной вытяжки, но для которого удавалось достигнуть лишь значения $\lambda=25$ [8] (вытяжка закристаллизованного расплава). В последнем случае площадь поперечного сечения $\left(10^{-7} \mathrm{~m}^{2}\right)$ на два десятичных порядка превышала площадь поперечного сечения образцов, исследованных в настоящей работе. Это и позволило провести измерения истинной деформации при использовании щупового датчика, закрепленного на базе образца с $\lambda=25$. Такая существенная разница между значениями $E$ для ПЭ с $M \sim 10^{5}$ и СВМПЭ с $M=3 \cdot 10^{6} \mathrm{~g} / \mathrm{mol}$ свидетельствует о формировании более жесткого „каркаса“ в образцах на основе СВМПЭ из цепных молекул, длина которых на один десятичный порядок превышает длину цепей высокомолекулярного ПЭ с $M \sim 10^{5}$.

С целью определения наиболее адекватной аналитической модели описания экспериментальных данных, представленных на рис. 1, позволяющей осуществлять прогнозирование величины $E$ в зависимости от $l_{0}$, данные рис. 2 были проанализированы при использовании различных функций, обеспечиваемых графической программой Origin. Результаты проведенного анализа показали, что наиболее корректно зависимость $E\left(l_{0}\right)$ может быть описана с помощью следующего эмпирического уравнения (1):

$$
E=A_{2}+\left(A_{1}-A_{2}\right) /\left(1+\exp \left[\left(l_{0}-x_{0}\right) / \Delta l_{0}\right]\right),
$$

где $A_{1}=-4797.5 \mathrm{GPa}, A_{2}=242.48 \mathrm{GPa}, x_{0}=-124.79 \mathrm{~mm}$ и $\Delta l_{0}=37.97 \mathrm{~mm}$ - константы, $l_{0}$ - база образца. В соответствии с уравнением (1), прогнозируемые значения $E$ при $l_{0}=100,200$ и $500 \mathrm{~mm}$ составляют 229.0, 241.5 и $242.5 \mathrm{GPa}$ соответственно. Это означает, что при $l_{0}=200 \ldots 500 \mathrm{~mm}$ происходит насыщение величины $E$ с выходом на плато $A_{2}=242.5 \mathrm{GPa}$, и дальнейшее увеличение $l_{0}$ с целью корректного измерения $E$ представляется нецелесообразным. Отметим, что значение $E=225 \mathrm{GPa}$, измеренное в настоящей работе при максимальном значении $l_{0}=80 \mathrm{~mm}$, ниже прогнозируемого значения $E=A_{2}$ всего лишь на $7 \%$. Такая разница представляется допустимой. Следовательно, длина рабочей 
части образца $l_{0}=80 \mathrm{~mm}$ может являться достаточной для корректного измерения модуля упругости ультраориентированных сверхпрочных материалов с малым поперечным сечением без использования датчика деформации, в особенности при ограниченном количестве необходимых для измерений образцов и при проведении статистического анализа большого массива измерений (например, пятидесяти).

Необходимо также отметить, что экспериментальное значение $E=225 \mathrm{GPa}$, измеренное при $l_{0}=80 \mathrm{~mm}$, является очень высоким, так как оно сопоставимо с экспериментальными и теоретическими значениями $E$ для предельно ориентированных материалов на основе СВМПЭ и модельной цепочки ПЭ, опубликованными в литературе, которые изменяются в широком интервале $E$ от 200 до $340 \mathrm{GPa}$ [9-12]. Следовательно, использование метода многоступенчатой зонной вытяжки, обеспечивающее достижение очень высоких значений $\lambda=120$, приводит к созданию сверхвысокомодульных пленочных мононитей на основе СВМПЭ с достижением значений $E$, близких к теоретическим.

\section{4. Заключение}

Показана высокая эффективность метода многостадийной ориентационной зонной вытяжки для получения сверхвысокомодульных ультраориентированных пленочных нитей СВМПЭ со степенью вытяжки $\lambda=120$ с достижением значения $E$ не менее $225 \mathrm{GPa}$, соизмеримого с теоретическими значениями $E$ для одиночной цепи ПЭ. Показано, что модуль упругости $E$ сверхориентированного СВМПЭ может быть корректно определен при использовании деформации, измеренной между зажимами разрывной машины при базе образца не менее $80 \mathrm{~mm}$. Исследована зависимость $E$ от $l_{0}$ для данных образцов. Получено эмпирическое уравнение, позволяющее прогнозировать величину модуля упругости $E$ в зависимости от расстояния между зажимами $l_{0}$. В соответствии с проведенным прогнозом установлено, что насыщение значения $E$, составляющее $E=242 \mathrm{GPa}$, происходит при $l_{0}=200 \mathrm{~mm}$.

\section{Список литературы}

[1] V.A. Marikhin, L.P. Myasnikova. Structural basis of highstrength high-modulus polymers. In: Oriented Polymer Materials / Ed. S. Fakirov. Huthig \& Wepf Verlag-Zug, Heidelberg (1996). P. 38-98.

[2] Yu.M. Boiko, V.A. Marikhin, L.P. Myasnikova, O.A. Moskalyuk, E.I. Radovanova. J. Mater. Sci. 52, 1727 (2017).

[3] Ю.М. Бойко, В.А. Марихин, Л.П. Мясникова, О.А. Москалюк, Е.И. Радованова. ФТТ 58, 2065 (2016).

[4] DSM info Issued: 20-09-2013 Page: 1/1 Ref.: dtm ya001.

[5] US Patent 4, 422, 993. Process for the preparation of filaments of high tensile strength and modulus. P. Smith, P.J. Lemstra. Ser. N 06/162,449, filling date June 24,1980 , issued date December 27, 1983.
[6] ГОСТ 10213.2-2002. Волокно штапельное и жгуты химические. Методы определения разрывной нагрузки и удлинения при разрыве.

[7] А.А. Аскадский. Деформация полимеров. Химия, М. (1973). $448 \mathrm{c}$.

[8] Yu.M. Boiko, I.G. Kuznetsova, S.A. Unezheva, V.V. Kovriga, L.A. Gann. Mech. Comp. Mater. 29, 1 (1993).

[9] V.A. Marikhin, L.P. Myasnikova, Yu.M. Boiko, E.M. Ivan'kova, E.I. Radovanova, P.N. Yakushev. Role of Reactor Powder Morphology in Producing High-strength High-modulus UHMWPE Fibres. In: Reactor Powder Morphology / Eds. L. Myasnikova, P. Lemstra. Nova Publishers, Hauppauge, NY. ISBN: 978-1-61668-592-8. Ch. 10. (2011). P. 235-294.

[10] D. Sawai, K. Nagai, K.M. Inato, T. Ohama, T. Kanamoto. J. Polym. Sci. B 44, 153 (2006).

[11] D.S. Boudreaux. J. Polym. Sci. B 11, 1285 (1973).

[12] B. Crist, J. Oddershede, J.R. Sabin, J.W. Perram, M.A. Ratner J. Polym. Sci. B 22, 881 (1984).

Редактор К.В. Емцев 